From the Departments of Obstetrics and Gynecology, Radiology, Rheumatology, Electrical Engineering, Orthopedic Surgery, Medical Oncology, and the Multidisciplinary Breast Center, Leuven Kanker Instituut, Katholieke Universiteit Leuven, University Hospitals KU, Leuven, Belgium.

Submitted November 16, 2007: accepted February 21, 2008; published online ahead of print at www.jco.org on May 12, 2008

L.M., S.P., and K.V. contributed equally to this work

Authors' disclosures of potential conflicts of interest and author contributions are found at the end of this article.

Corresponding author: Patrick Neven, MD, Department of Obstetrics and Gynecology, Division of Gynecological Oncology, University Hospital Gasthuisberg, Herestraat 49, 3000 Leuven, Belgium; e-mail: Patrick.Neven@ uz.kuleuven.ac.be.

(C) 2008 by American Society of Clinical Oncology

0732-183X/08/2619-1/\$20.00

DOI: 10.1200/JCO.2007.15.4005

\title{
Prospective Study to Assess Short-Term Intra-Articular and Tenosynovial Changes in the Aromatase Inhibitor- Associated Arthralgia Syndrome
}

Leilani Morales, Steven Pans, Kilian Verschueren, Ben Van Calster, Robert Paridaens, Rene Westhovens, Dirk Timmerman, Luc De Smet, Ignace Vergote, Marie-Rose Christiaens, and Patrick Neven

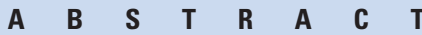

\section{Purpose}

Arthralgia is an adverse class effect of aromatase inhibitors (Als). To date, its exact mechanism remains unclear. The purpose of this study was to investigate the changes in clinical rheumatologic features and magnetic resonance imaging (MRI) of hands and wrists in Al and tamoxifen users.

\section{Patients and Methods}

This is a prospective single-center study including 17 consecutive postmenopausal patients with early breast cancer receiving either tamoxifen $(n=5)$ or an $\mathrm{Al}(n=12)$. At baseline and after 6 months, patients filled in a rheumatologic history questionnaire and a rheumatologic examination including a grip strength test was done. At the same time points, MRI of both hands and wrists was performed. The primary end point was tenosynovial changes from baseline on MRI. Secondary end points were changes from baseline for morning stiffness, grip strength, and intra-articular fluid on MRI. Wilcoxon signed ranks was used to test changes from baseline and the Spearman correlation coefficient to assess the association between rheumatologic and MRI changes from baseline.

\section{Results}

At 6 months, patients on $\mathrm{Al}$ had a decrease in grip strength $(P=.0049)$ and an increase in tenosynovial changes $(P=.0010)$. The decrease in grip strength correlated well with the tenosynovial changes on $\mathrm{MRI}(P=$.0074). Only minor changes were seen in patients on tamoxifen. Al users reported worsening of morning stiffness and showed an increase in intra-articular fluid on MRI.

\section{Conclusion}

The functional impairment of hands in the Al-associated arthralgia syndrome is characterized by tenosynovial changes on MRI correlating with a significant decrease in hand grip strength.

\section{J Clin Oncol 26. (C) 2008 by American Society of Clinical Oncology}

\section{INTRODUCTION}

Endocrine therapy remains the most important systemic treatment in women with a hormone receptor-positive breast cancer. Recent clinical trials have shown that the nonsteroidal aromatase inhibitors (AIs), anastrozole and letrozole and the steroidal AI, exemestane, are superior in disease-free survival compared with tamoxifen either as monotherapy or when used sequentially after tamoxifen therapy. ${ }^{1-6} \mathrm{It}$ is therefore expected that in the coming years, the use of AIs will dramatically increase worldwide, especially for the adjuvant treatment of postmenopausal women with endocrine sensitive early breast cancer. ${ }^{7-9}$

It has been generally accepted that endocrine treatments, such as AIs and tamoxifen, are reasonably well tolerated by most patients. Adverse effects of AIs such as osteoporosis and osteoporotic fractures have been extensively evaluated ${ }^{10-13}$ and addressed. ${ }^{14}$ Less is known about AI-associated musculoskeletal pain, which up to now has been nonspecifically termed arthralgia, although it may be a syndrome broader than arthralgia alone. The link between AI therapy and arthralgia was first reported 7 years ago. ${ }^{15}$ Later, all major adjuvant trials comparing AIs with either tamoxifen or a placebo reported more musculoskeletal complaints under AIs, mostly mild to moderate in severity and with a modest impact on daily functioning. However, because of the lack of uniform assessment, relying on formal toxicity reporting by patients without corresponding rheumatologic evaluation, the true prevalence and severity of this adverse effect may be underestimated. 
Recently, AI-associated musculoskeletal pain was shown to be more prevalent and important than previously described in clinical trials ${ }^{16}$ and the existence of an AI-associated arthralgia syndrome has been recognized by clinicians. ${ }^{17-18}$ Some patients experience incapacitating symptoms limiting treatment compliance $e^{15,19}$ and thus warranting proper management. The exact mechanism of the AI-associated arthralgia syndrome remains unclear, but is believed to be related to the deep estrogen deprivation induced by AIs.

We previously described in a retrospective case series the impact of severe arthralgia on normal daily functioning and the tenosynovial changes on ultrasound and MRI. ${ }^{19}$ We hypothesized that the tendon sheath abnormalities as seen with MRI were the primary cause of the reported musculoskeletal pain. Considering the limited data that is available, we prospectively investigated the changes in clinical rheumatologic features and MRI of hands and wrist in AI and tamoxifen users.

\section{PATIENTS AND METHODS}

We conducted a prospective single-center study in the University Hospital Gasthuisberg (Leuven, Belgium). Consecutive postmenopausal women with early breast cancer scheduled to start first-line adjuvant endocrine therapy with any of the third generation AIs (anastrozole, letrozole, or exemestane) or tamoxifen were included in this study. The decision to start on tamoxifen or an AI was based on our institution's in-house protocol for use of either therapy and there was no self selection of patients based on age or underlying joint symptoms. The patients completed a self-administered demographic data and rheumatologic history questionnaire which was specifically designed for this study. This questionnaire inquired on rheumatic complaints in the past 5 years such as morning stiffness, joint pains, limitation of movements, muscle pains, and numbness of hands. All were to be answered by yes/no and if the answer was yes, a more detailed history was elicited. A single rheumatologist (K.V.), who was blinded to the endocrine treatment and MRI findings, performed clinical examination of both hands, which included careful assessment of joint and tendon abnormalities and functional evaluation by a hand grip test. The latter was achieved by asking the subject to squeeze the balloon of a modified sphygmomanometer three times with maximal force and recording the average value of three trials for each hand. Higher scores (reported in $\mathrm{kPa}$ ) reflect better grip strength. The findings were recorded in a rheumatologic clinical evaluation form specifically designed for this study. The following parameters were taken into account: generalized musculoskeletal pain, morning stiffness, hand stiffness/pain/paresthesia, flexor tendon nodules/contractures/pain/trigger phenomenon, osteoarthritis (OA), and synovitis. OA was defined as presence of Heberden and Bouchard nodules while morning stiffness was taken into account if lasting at least 30 minutes.

MRI of both hands and wrists was performed in each patient at 1.5 Tesla (Siemens Sonata, Erlangen, Germany) using a dedicated four-channel wrist coil. Images were acquired by using a T2-weighted sequence with fat suppression and a T1-weighted sequence in a transverse and coronal plane. In order to determine tenosynovial and synovial contrast enhancement and to determine the amount of fluid in the tendon sheath and in the joints of hand and wrist, follow-up MRI was performed after gadolinium contrast administration using T1-weighted and T2-weighted sequence with fat suppression. All images were interpreted by the same experienced musculoskeletal radiologist (S.P.) who was blinded to the treatment patients were receiving and to other clinical data.

Both clinical and radiologic follow-up investigations were repeated after 6 months of therapy or earlier when patients considered stopping treatment prematurely because of severe joint complaints. The study was conducted according to the guidelines for clinical studies described in the Declaration of Helsinki. ${ }^{20}$ The protocol was approved by the Ethical Committee for Clinical Studies of the University Hospitals Leuven, Belgium, before commencement of the study and all participants provided written informed consent.

\section{Statistical Analysis}

The repeat rheumatologic examination and MRI at 6 months took into account all baseline findings. The rheumatologic examination results after 6 months therapy are reported as either a new onset of symptoms or worsening of a pre-existing symptom from baseline for each patient. Scores could be -1 (improvement), 0 (no change), or 1 (worsening). MRI results are reported as number of images that showed the pathology over the total of images taken (there is one image for each hand and thus two images per patient). MRI results on intra-articular fluid and tenosynovial changes were scored as 0 (no difference), -1 (improvement of pre-existing findings or disappearance), or +1 (worsening of pre-existing findings or new onset). A combined score was constructed by summing the scores of both hands. This score can equal -2 (improvement for both hands), -1 (improvement for one hand, no change for the other), 0 (no change for both hands, or improvement for one hand and worsening for the other), +1 (worsening for one hand, no change for the other), and +2 (worsening for both hands).

The primary end point was tenosynovial changes from baseline on MRI. Tenosynovial changes were defined as an increase in the amount of fluid in the tendon sheath or thickening and enhancement of the tendon sheaths. Secondary end points were changes from baseline for morning stiffness, grip strength, and intra-articular fluid on MRI. Descriptive data are provided and statistical analysis was performed using the Wilcoxon signed ranks to test changes from baseline and the Spearman correlation coefficient was used to detect associations between grip strength and MRI changes. Statistical tests were always two-sided.

\section{RESULTS}

All 17 patients completed all the planned investigations and are included in this report. Baseline demographic and medical characteristics are described in Table 1. Mean age was 65 years (range, 54 to 74 years) and patients were on average 14 years past menopause (range, 1 to 39 years). Tamoxifen users were younger and had a higher mean body mass index compared to AI users. Endocrine therapies were the following: AIs in 12 patients (letrozole, $\mathrm{n}=5$; exemestane, $\mathrm{n}=4$; anastrozole, $n=3$ ) and tamoxifen in five patients. There were four patients who received adjuvant chemotherapy before endocrine therapy, two patients in the AI group, and two in the tamoxifen group. The baseline rheumatologic findings showed that pre-existing degenerative joint disease was already present in 12 patients and morning stiffness was reported by three patients. Baseline mean grip strength was comparable in each group. On baseline MRI, there was fluid accumulation seen in the joints of three AI users and in one of the tamoxifen patients. Tenosynovial abnormalities were seen in three of the AI users and in one of the tamoxifen patients.

Taking into account clinical examination as well as patient complaints, changes from baseline were seen in 11 of 12 patients on AI and in three of five patients on tamoxifen. Five AI patients experienced increased morning stiffness and no one had improvement of morning stiffness, but this change was not statistically significant $(P=.0625)$; larger numbers of patients will be required to confirm this finding. No one had improvement for morning stiffness. It is noteworthy that none of the tamoxifen patients experienced increased or new onset morning stiffness. Table 2 presents that after 6 months AI users had a marked decrease in grip strength which was statistically significant $(P=.0049)$. AI users were 2.08 times more likely to have a decrease in grip strength (10 of 12) than tamoxifen users (two of five; relative risk $=2.08$; 95\% CI, 0.93 to 7.48, Cox-Hinkley-Miettinen-Nurminen method).

On MRI follow-up, 11 AI patients had worsening of pre-existing changes or new onset of any pathology of joints or tendons, eight of 


\begin{tabular}{|c|c|c|}
\hline Treatment & $\mathrm{Al}$ & Tam \\
\hline No. of patients & 12 & 5 \\
\hline Mean age, years & 68 & 59 \\
\hline Range & $58-74$ & $54-68$ \\
\hline Mean BMI, kg/m² & 22 & 25 \\
\hline Range & $21-23$ & $21-45$ \\
\hline Mean years past menopause & 16 & 10 \\
\hline Range & $1-39$ & $1-26$ \\
\hline Previous intake of HRT & 6 & 3 \\
\hline \multicolumn{3}{|l|}{ Clinical tumor size, $\mathrm{cm}$} \\
\hline$<2$ & 5 & 1 \\
\hline$\geq 2$ to 5 & 7 & 3 \\
\hline$>5$ & 0 & 1 \\
\hline \multicolumn{3}{|l|}{ Pathologic nodal status } \\
\hline Negative & 7 & 1 \\
\hline Positive & 5 & 4 \\
\hline \multicolumn{3}{|l|}{ Adjuvant chemotherapy } \\
\hline FEC regimen & 0 & 1 \\
\hline Sequential taxane and $\mathrm{FEC}$ & 2 & 1 \\
\hline \multicolumn{3}{|l|}{ Rheumatologic findings } \\
\hline Osteoarthritis* & 9 & 3 \\
\hline Morning stiffnesst & 2 & 1 \\
\hline Mean grip strength, $\mathrm{kPa}$ & 44.5 & 40.7 \\
\hline Range & 23.3-62.3 & $30.3-51.0$ \\
\hline \multicolumn{3}{|l|}{ MRI findings } \\
\hline Intra-articular fluid & 3 & 1 \\
\hline Tenosynovial abnormalities & 3 & 1 \\
\hline \multicolumn{3}{|c|}{$\begin{array}{l}\text { Abbreviations: Al, aromatase inhibitor; Tam, tamoxifen; BMI, body mass } \\
\text { index; HRT, hormone replacement therapy; FEC, fluorouracil, epirubicin, and } \\
\text { cyclophosphamide; MRI, magnetic resonance imaging. } \\
{ }^{*} \text { Osteoarthritis defined as numbers of patients with Heberden and } \\
\text { Bouchard nodules. } \\
\text { †Morning stiffness defined as number of patients reporting this at least lasting } \\
\text { for } 30 \text { minutes. } \\
\neq \text { Grip strength reported as the mean and range of both hands. }\end{array}$} \\
\hline
\end{tabular}

them in both hands while one patient had an improvement shown as a decrease in intra-articular fluid. Half of the tamoxifen patients with a follow-up MRI had no changes at all. The changes appearing on tamoxifen were less pronounced than when they appeared on AIs. Although the increase in intra-articular fluid after 6 months in AI patients was not statistically significant $(P=.0625)$, significant tenosynovial changes were evident as presented in Table $3(P=.0010)$. The risk of having score higher than 0 (worsening) was 3.67 times higher for patients on AI (11 of 12) than for patients on tamoxifen (one of four; relative risk $=3.67 ; 95 \% \mathrm{CI}, 1.22$ to 21.2 ). Table 4 demonstrates tenosynovial changes in relation with grip strength. It shows that worsening of tenosynovial changes on follow-up was strongly related
Table 3. Magnetic Resonance Imaging Tenosynovial Changes After 6 Months of Antihormone Therapy

\begin{tabular}{lcccc}
\hline & & \multicolumn{3}{c}{ Change } \\
\cline { 3 - 5 } Therapy & No. of Patients & $0^{*}$ & $1 \dagger$ & $2 \neq$ \\
\hline Al & 12 & 1 & 3 & 8 \\
Tam & 4 & 3 & 0 & 1 \\
\hline
\end{tabular}

NOTE. Wilcoxon signed ranks test to test for Al users, $P=.0010$

Abbreviations: Al, aromatase inhibitor; Tam, tamoxifen.

*0 indicates no change for both hands, or improvement for one hand and worsening for the other.

$\dagger+1$ indicates worsening for one hand, no change for the other

$\ddagger+2$ indicates worsening for both hands

to a higher decrease in grip strength (Spearman correlation $=-0.64$; $P=.0074)$. There was no association seen between intra-articular fluid and decreased grip strength (Spearman correlation $=0.11 ; P=.6852$ ).

Typical MRI changes from baseline to after 6 months of therapy in two patients on an AI are shown in Figures 1 and 2. These images show an enhancement of the synovium, increase in intra-articular fluid, and tenosynovial changes with contrast enhancement and fluid in the tendon sheath.

Two patients discontinued treatment early before the 6 months re-evaluation due to severe arthralgia. One was on an AI without prior adjuvant chemotherapy and the other was on tamoxifen with prior adjuvant chemotherapy. In the first patient who stopped exemestane after 4 months, carpal tunnel syndrome was evident on clinical rheumatologic examination. MRI showed bilateral signs of tenosynovial changes of flexor tendon sheaths and enhanced uptake of contrast at the level of the carpal tunnel on the right side. For the other patient, unfortunately, the repeat MRI was performed late, after switch from tamoxifen to anastrozole, and must be considered unassessable within the frame of this study. It is, however, noteworthy that an important decrease in grip strength was seen in both patients at the moment of discontinuation of treatment compared with baseline.

\section{DISCUSSION}

To our knowledge, this is the first prospective study investigating the AI-associated arthralgia syndrome by means of a standard rheumatologic evaluation including functional assessment and the use of MRI to demonstrate the anatomic substrate. This study showed that after 6 months, AIs induce more pain and functional impairment of the hands and wrists than tamoxifen. The complaints of the affected patients are compatible with the reported AI-associated arthralgia

\begin{tabular}{|c|c|c|c|c|c|c|}
\hline $\mathrm{Al}$ & 12 & -16.28 & $-9.51^{*}$ & 1 & 10 & $-73.4-4.84$ \\
\hline \multicolumn{7}{|c|}{$\begin{array}{l}\text { NOTE. The total \% change is computed as the percentage change in total strength at } 6 \text { months (strength left hand at } 6 \text { months plus strength right hand at } 6 \text { months } \\
\text { relative to baseline (strength left hand at baseline plus strength right hand at baseline). } \\
\text { Abbreviations: Al, aromatase inhibitor; Tam, tamoxifen. } \\
\text { "Wilcoxon signed rank test, } P=.0049 \text {. }\end{array}$} \\
\hline
\end{tabular}




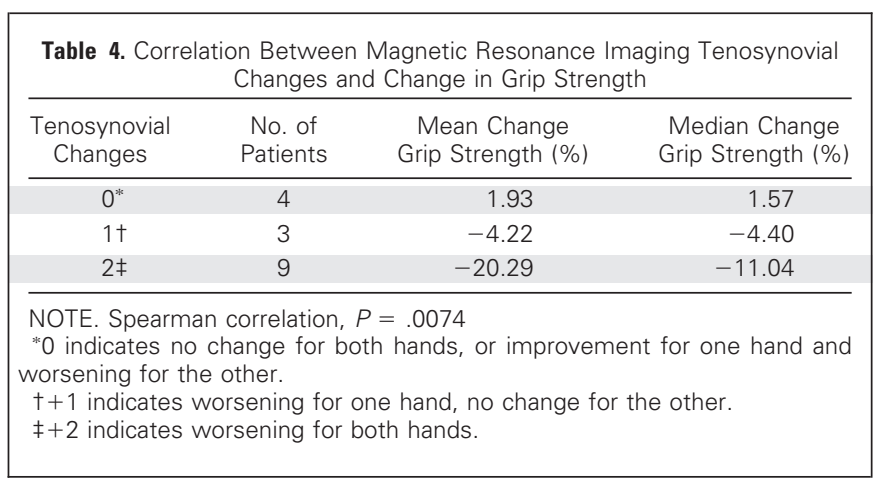

syndrome, consisting of early morning stiffness and pain, usually symmetric, typically in the hands but frequently also in other joints.

Sequential rheumatologic examination showed a marked decrease in hand grip strength in AI users compared to tamoxifen users. New onset or worsening of the highly prevalent pre-existing musculoskeletal disorders was also more frequent in AI users; however, there was no clear increase in clinically detectable synovitis. MRI after 6 months showed worsening of articular and tenosynovial changes, the latter strongly correlating with a greater decrease in grip strength. This suggests that tenosynovial and articular changes as seen on MRI identify the anatomic substrate for the hand pain and disability which characterize the AI-associated arthralgia syndrome. It also provides a plausible explanation for the increased incidence of carpal tunnel syndrome in patients treated with AIs, as fluid accumulation and thickening of the tendon sheaths of the wrist may lead to compression of the median nerve.

In view of the high background of nonspecific muscle and joint aches in women affected by breast cancer, it is important to perform a careful clinical and radiologic evaluation at baseline and at fixed time during follow-up. This design allows using each patient as her own control so that our results may be considered as unbiased since we report changes induced by treatment rather than mere prevalence of symptoms or radiologic findings. The scoring we adapted for morning stiffness took into account baseline findings where scores could be -1 (improvement), 0 (no change), or 1 (worsening). This allows for a qualitative approach and specific detection of all possible changes from baseline to 6 months of therapy. We included the hand grip test as an objective, reproducible, and clinically relevant tool for the quantitative assessment of hand and wrist disability. ${ }^{21-24}$ Although sophisticated technology of assessment is not necessarily superior to clinical evaluation, ${ }^{25}$ we included MRI in this study because it is by far the most sensitive and reproducible imaging technique to explore clinical and subclinical joint problems in patients with arthralgia or early rheumatoid changes. ${ }^{26-28}$ The analysis and scoring of MRI images are similar to a recent publication ${ }^{28}$ where pathologies were scored as 0 or 1 (absent or present). However, for our study, we adapted a more detailed scoring system for both the rheumatologic examination and MRI, always taking into account the baseline findings, given the importance of baseline findings in this population. This scoring system allowed us to detect all possible changes in our variables from baseline and as such, it also enabled us to use the Wilcoxon signed ranks to test all changes from baseline to 6 months of therapy. A potential weakness in our study design was MRI reproducibility as we lack data on interand intraobserver variability in image interpretation. However, all
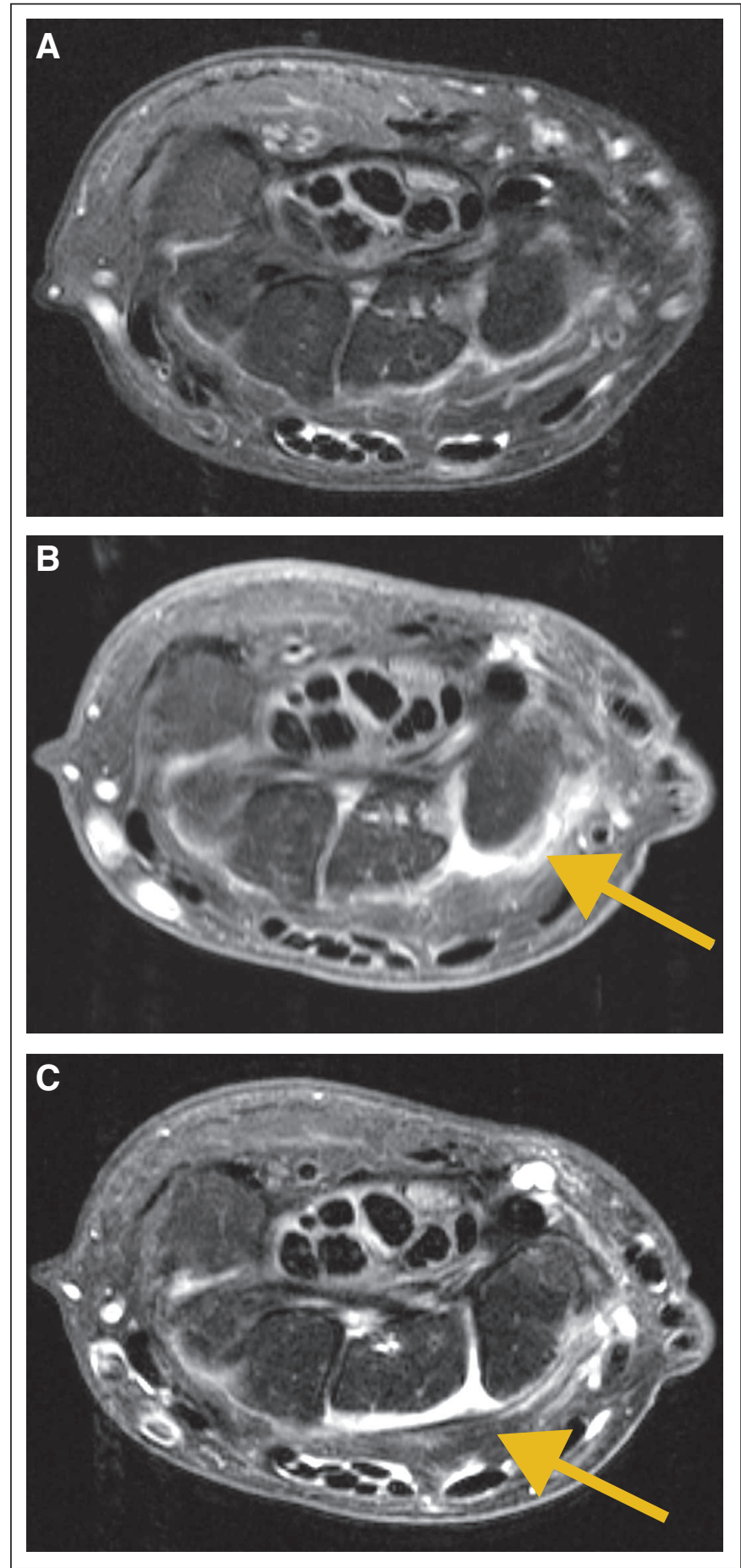

Fig 1. (A) Baseline T2-weighted image with fat suppression of the wrist in a transverse plane. ( $B, C)$ Follow-up magnetic resonance imaging after 6 months of therapy. (B; arrow) T1-weighted image with fat suppression shows an enhancement of synovium of wrist. (C; arrow) The T2-weighted image with fat suppression demonstrates an increase of the amount of intra-articular fluid in wrist.

images were assessed by the same musculoskeletal radiologist (C.E.) who was blinded to the treatment patients were receiving and to the other clinical data. Furthermore, the prospective design of this study with baseline and follow-up images together with the excellent correlation between the MRI and clinical findings support our conclusion. 

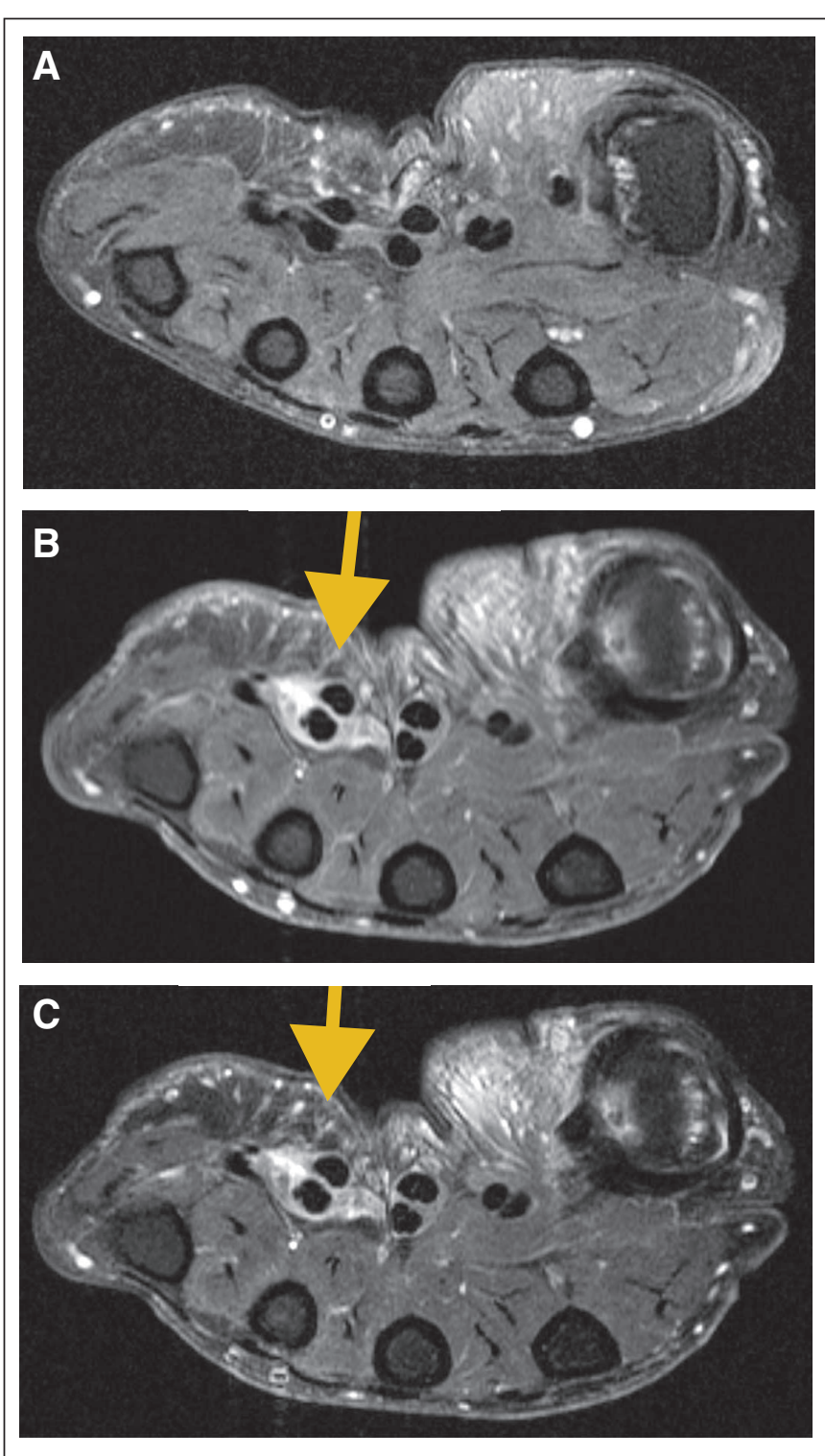

Fig 2. (A) Baseline fat suppressed $T 2$-weighted image of the hand. $(B, C)$ Follow-up magnetic resonance imaging after 6 months of therapy. (C; arrow) Severe tenosynovial changes of the deep and superficial digital flexor tendon in the fourth row were diagnosed on the T2-weigthed image. (B; arrow) An enhancement of the tendon sheath was depicted on the postcontrast fat suppressed T1-weighted image.

Other factors may influence the onset, severity, and the evolution of the AI-associated arthralgia syndrome. There is an association with prior chemotherapy, especially taxanes. ${ }^{16}$ It has also been reported by Buzdar et $\mathrm{al}^{29}$ that AI-associated arthralgia occurs earlier and is more frequent in younger postmenopausal women. As seen in the baseline demographics of our study, the mean age of AI users was 68 years. Our results in this elderly group therefore strengthen our findings.

Although arthralgia did not severely affect compliance in all major AI trials, such as Arimidex, Tamoxifen Alone or in Combination, ${ }^{29}$ in our clinical experience ${ }^{19}$ and those of others, ${ }^{15}$ joint stiffness/ pain does interfere with a woman's daily functioning and general well being. This may affect treatment compliance ${ }^{30-32}$ especially when symptoms typically in the hands are incapacitating. ${ }^{15,19}$ Nonsteroidal anti-inflammatory drugs may alleviate musculoskeletal pain ${ }^{16,19}$ but in our experience, only a minority of patients responds to this treatment. It is important to note that in this setting, inflammatory parameters such as C-reactive protein and erythrocyte sedimentation rate are not elevated, suggesting the absence of a systemic inflammatory process. As long as there is no evidence for an inflammatory etiology, the routine use of nonsteroidal anti-inflammatory drugs to alleviate symptoms does not seem justified.

The pathogenesis of the AI-associated arthralgia syndrome is supposed to be related to deep estrogen deprivation induced by the potent third generation AIs. There are numerous reports exploring a possible link between estrogen deprivation, joint disorders, and pain. In 1925, Cecil and Archer described menopausal arthritis. ${ }^{33}$ The prevalence of joint pain and aches indeed increases progressively with age in women, reaching a maximum in the 50 to 59 years age group, suggesting an association with menopause. Also, in some countries where postmenopausal estrogen levels are low, arthralgia is reported to be the predominant somatic symptom of menopause as it is in parts of Asia. ${ }^{34}$

While arthralgia merely describes symptoms without taking into account objective joint findings, we should remember that the most common forms of articular pathologies also have their highest incidence in postmenopausal women such as OA and rheumatoid arthritis. Although there may be a protective effect of estrogen use in radiographic OA, a consistent effect on the occurrence or disease activity of rheumatoid arthritis has not been shown. ${ }^{35}$

Because estrogen can exert a direct antinociceptive effect on the CNS, ${ }^{18,36-38}$ one may hypothesize that estrogen deprivation could lead to enhanced pain sensation at a peripheral level: the absence of circulating estrogens at the level of estrogen receptors in joint tissue may also induce as yet unknown biochemical and pathophysiological changes leading to debilitating joint pain. It is clear that additional studies are needed to evaluate other mechanisms behind AI-associated arthralgia. Some endocrine disorders may have musculoskeletal manifestations similar to patients with AI-associated arthralgia. In particular, diabetes mellitus may present with a variety of associated rheumatic manifestations in the musculoskeletal system including limited joint mobility, trigger finger, and carpal tunnel syndrome. ${ }^{39}$ Furthermore, it is our impression that clinical and radiologic findings in the joints of AI users resemble findings seen in hands of diabetic patients which shows an increase in fluid and tenosynovial thickness on MRI. ${ }^{40}$ However, the exact mechanisms in which endocrine disorders lead to musculoskeletal manifestations need to be further investigated. It may be important that future research will explore common metabolic pathways between diabetes mellitus and AIassociated arthralgia.

In conclusion, we have shown that more than half of the postmenopausal patients with breast cancer scheduled to receive adjuvant endocrine therapy had some form of pre-existing musculoskeletal disorder. New onset or worsening of pre-existing symptoms was more frequent and more pronounced in AI than in tamoxifen users. AI users were more likely to develop a decrease in grip strength and an increase in tenosynovial changes. Our results are hypothesis generating. The excellent correlation between clinical and MRI findings provides an objective substrate for the subjective complaints of the AI-associated arthralgia syndrome. 


\section{AUTHORS' DISCLOSURES OF POTENTIAL CONFLICTS} OF INTEREST

The author(s) indicated no potential conflicts of interest.

\section{AUTHOR CONTRIBUTIONS}

Conception and design: Leilani Morales, Steven Pans, Kilian

Verschueren, Robert Paridaens, Rene Westhovens, Patrick Neven

Provision of study materials or patients: Robert Paridaens, Marie-Rose Christiaens, Patrick Neven
Collection and assembly of data: Leilani Morales, Steven Pans, Kilian Verschueren, Robert Paridaens, Rene Westhovens, Dirk Timmerman, Luc De Smet, Ignace Vergote, Marie-Rose Christiaens, Patrick Neven Data analysis and interpretation: Leilani Morales, Steven Pans, Kilian Verschueren, Ben Van Calster, Robert Paridaens, Rene Westhovens, Dirk Timmerman, Luc De Smet, Ignace Vergote, Marie-Rose Christiaens, Patrick Neven

Manuscript writing: Leilani Morales, Steven Pans, Kilian Verschueren, Robert Paridaens, Rene Westhovens, Patrick Neven Final approval of manuscript: Leilani Morales, Steven Pans, Kilian Verschueren, Ben Van Calster, Robert Paridaens, Rene Westhovens, Dirk Timmerman, Luc De Smet, Ignace Vergote, Marie-Rose Christiaens, Patrick Neven

\section{REFERENCES}

1. The ATAC Trialists' Group: Results of the ATAC (Arimidex, Tamoxifen Alone or in Combination) trial after completion of 5 years' adjuvant treatment for breast cancer. Lancet 365:60-62, 2005

2. Goss PE, Ingle JN, Martino S, et al: Efficacy of letrozole extended adjuvant therapy according to estrogen receptor and progesterone receptor status of the primary tumor: National Cancer Institute of Canada Clinical Trials Group MA. 17. J Clin Oncol 25:2006-2011, 2007

3. Coombes RC, Hall E, Gibson LJ, et al: A randomized trial of exemestane after two to three years of tamoxifen therapy in postmenopausal women with primary breast cancer. $N$ Engl J Med 350:1081-1092, 2005

4. Coombes RC, Kilburn LS, Snowdom CF, et al: Survival and safety of exemestane versus tamoxifen after 2-3 years' tamoxifen treatment (Intergroup Exemestane Study): A randomized controlled trial. Lancet 369:559-570, 2007

5. The Breast International Group (BIG) 1-98 Collaborative Group: A comparison of letrozole and tamoxifen in postmenopausal women with early breast cancer. N Engl J Med 353:2747-2757, 2005

6. Coates AS, Keshaviah A, Thürlimann B, et al: Five years of letrozole compared with tamoxifen as initial adjuvant therapy for postmenopausal women with endocrine-responsive early breast cancer: Update of study BIG 1-98. J Clin Oncol 25:486-492, 2007

7. Rabaglio M, Aebi S, Castiglione-Gertsch M: Controversies of adjuvant endocrine treatment for breast cancer and recommendations of the $2007 \mathrm{St}$ Gallen conference. Lancet Oncol 8:940-949, 2007

8. Poole $R$, Paridaens $R$ : The use of thirdgeneration aromatase inhibitors and tamoxifen in the adjuvant treatment of postmenopausal patients with hormone-dependent breast cancer: Evidence based review. Curr Opin Oncol 19:564-572, 2007

9. Morales L, Neven P, Paridaens R: Choosing between an aromatase inhibitor and tamoxifen in the adjuvant setting. Curr Opin Oncol 17:559-565, 2005

10. Goss PE: Risk versus benefits in the clinical application of aromatase inhibitors. EndocrineRelated Cancer 6:325-332, 1999

11. Gradishar W: Safety considerations of adjuvant therapy in early breast cancer in postmenopausal women. Oncology 69:1-9, 2005

12. Cella D, Fallowfield LJ: Recognition and management of treatment-related side effects for breast cancer patients receiving adjuvant endocrine therapy. Breast Cancer Res Treat 107:167-180, 2008

13. Coleman RE, Banks LM, Girgis SI, et al: Skeletal effects of exemestane on bone-mineral density, bone biomarkers, and fracture incidence in postmenopausal women with early breast cancer participating in the Intergroup Exemestane Study (IES): A randomized controlled study. Lancet Oncol 8:119-127, 2007

14. Brufsky A. Management of cancer-treatmentinduced bone loss in postmenopausal women undergoing adjuvant breast cancer therapy: A Z-FAST update. Semin Oncol 33:S13- S17, 2006 (suppl 7)

15. Donnellan $P$, Douglas $S$, Cameron $D$, et al: Aromatase inhibitors and arthralgia. J Clin Oncol 19:2767, 2001

16. Crew KD, Greenlee H, Capodice J, et al: Prevalence of joint symptoms in postmenopausal women taking aromatase inhibitors for early-stage breast cancer. J Clin Oncol 25:3877-3883, 2007

17. Burstein HJ, Winer EP: Aromatase inhibitors and arthralgias: A new frontier in symptom management for breast cancer survivors. J Clin Oncol 25: 3797-3799, 2007

18. Felson D, Cummings S: Aromatase inhibitors and the syndrome of arthralgias with estrogen deprivation. Arthritis Rheum 52:2594-2598, 2005

19. Morales L, Pans S, Paridaens R, et al: Debilitating musculoskeletal pain and stiffness with letrozole and exemestane: Associated tenosynovial changes on magnetic resonance imaging. Breast Cancer Res Treat 104:87-91, 2007

20. World Medical Association: Review of the Declaration of Helsinki. http://www.wma.net

21. Nordenskiold U, Grimby G: Assessment of disability in women with rheumatoid arthritis in relation to grip force and pain. Disabil Rehabil 19:13-19, 1997

22. Kaegi C, Thibault MC, Giroux F, et al: The interrater reliability of force measurements using a modified sphygmomanometer in elderly subjects. Phys Ther 78:1095-1103, 1998

23. Thyberg I, Hass UA, Nordenskiold U, et al: Activity limitation in rheumatoid arthritis correlates with reduced grip force regardless of sex: The Swedish TIRA project. Arthritis Rheum 53:886-896, 2005

24. Allen KD, De Vellis RF, Renner JB, et al: Validity and factor structure of the AUSCAN osteoarthritis hand index in a community-based sample. Osteoarthritis Cartilage 15:830-836, 2007

25. Dequeker J, Esselens G, Westhovens R: Educational issues in rheumatology: The musculoskeletal examination: A neglected skill. Clin Rheumatol 26:5-7, 2007
26. Oneson SR, Scales LM, Erickson SJ, et al: MR imaging of the painful wrist. Radiographics 16:9971008, 1996

27. Mc Queen F, Stewart N, Crabbe F, et al: Magnetic resonance imaging of the wrist in early rheumatoid arthritis reveals a high prevalence of erosions at 4 months after symptom onset. Ann Rheum Dis 57:350-356, 1998

28. Calisir C, Aynasi A, Korkmaz C: The accuracy of magnetic resonance imaging of the hands and feet in the diagnosis of early rheumatoid arthritis. Joint Bone Spine 74:362-367, 2007

29. Buzdar A, on behalf of the ATAC Trialists Group: Clinical features of joint symptoms observed in the "Arimidex" Tamoxifen, Alone or in Combination (ATAC) trial. J Clin Oncol 24:15S, 2006 (suppl; abstr 551)

30. Partridge $A H$, LaFountain $A$, Taylor $B$, et al: Adherence with adjuvant anastrozole therapy among women with early breast cancer. Breast Cancer Res Treat 100:S186, 2006 (suppl 1; abstr 4044)

31. Chlebowski RT, Geller ML: Adherence to endocrine therapy for breast cancer. Oncology 7:1-9, 2006

32. Crean SM, Reynolds M, Motabar S, et al: Discontinuation rates of adjuvant letrozole or anastrozole in breast cancer patients in an electronic medical record database observational study. The 2007 ASCO Breast Cancer Symposium Proceedings, Chicago, IL, June 1-5, 2007 (abstr 229)

33. Cecil $\mathrm{RL}$, Archer $\mathrm{BH}$ : Arthritis of the menopause. JAMA 84:75-79, 1925

34. Ho SC, Chan SG, Yip YB, et al: Menopausal symptoms and symptom clustering in Chinese women. Maturitas 33:219-227, 1999

35. Cutulo M: Sex hormone adjuvant therapy in rheumatoid arthritis. Rheum Dis Clin North Am 26:881-895, 2000

36. Eckersell CB, Popper P, Micevych PE: Estrogen-induced alteration of $\mu$-opioid receptor immunoreactivity in the medial preoptic nucleus and medial amygdala. J Neurosci 18:3967-3976, 1998

37. Blomqvist $A$ : Sex hormones and pain: $A$ new role for brain aromatase? J Comp Neurol 423:549-551, 2000

38. Flores CA, Shughrue $P$, Petersen $S L$, et al: Sex related differences in the distribution of opioid receptor-like 1 receptor $\mathrm{mRNA}$ and colocalization with estrogen receptor mRNA in neurons of the spinal trigeminal nucleus caudalis in the rat. Neuroscience 118:769-778, 2003

39. Jacobs-Kosmin D, DeHoratius R: Musculoskeletal manifestations of endocrine disorders. Curr Opin Rheumatol 17:64-69, 2004

40. Khanna G, Polly F: MRI of diabetic cheiroarthropathy. AJR 188:W94-95, 2007

\section{Acknowledgment}

We thank the physicians, nurses, and secretaries who assisted us, and especially all the patients who kindly agreed to participate in this study. 